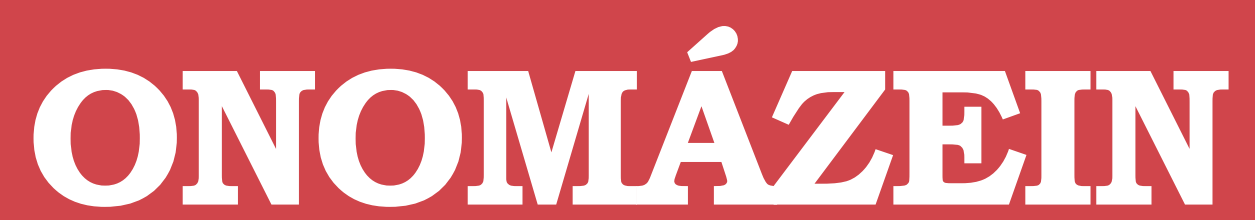

Revista semestral de lingüística, filología y traducción
PONTIFICIA UNIVERSIDAD

\title{
Más allá del aula: el papel de la Asociación Española Universitaria de Traductores e Intérpretes en Formación en la adquisición de competencias
}

Learning outside the classroom: the role of the Spanish Translation and Interpreting Students Association in the competence acquisition process

\section{Christian Olalla-Soler}

Universitat Autònoma de Barcelona

España 


\section{Resumen}

El objetivo del presente artículo es describir el papel que desempeña la Asociación Española Universitaria de Traductores e Intérpretes en Formación (AETI) en la formación de traductores e intérpretes.

Para ello, se exponen los resultados de un estudio empírico en el cual se compara la valoración de quince competencias del Libro banco. Título de Grado en Traducción e Interpretación (ANECA, 2004) entre 60 estudiantes socios de AETI y 60 estudiantes no socios de todos los cursos de la licenciatura y del grado en Traducción e Interpretación.

En los resultados generales se muestra que AETI funciona como complemento formativo al servir de fuente de información y de recursos para la formación de los estudiantes, ya que les aporta una serie de conocimientos y habilidades que no siempre son fáciles de adquirir en un aula.

Palabras clave: estudio empírico; formación de traductores e intérpretes; asociacionismo; adquisición de competencias.

\section{Abstract}

The main aim of this article is to describe the role of the Spanish Translation and Interpreting Students Association (AETI) in the translators and interpreters training.

To do so, an empirical study was carried out in which the evaluation of fifteen competences from the Libro blanco. Título de Grado en Traducción e Interpretación (ANECA, 2004) was compared between
60 students who are members of AETI and 60 who are not.

The general results show that AETI works as a training complement because it serves as a source of information and training resources for students. By being member of AETI, students acquire some knowledge and abilities that are not always easy to develop in class.

Keywords: empirical study; translator and interpreter training; associativity; competence acquisition. 


\section{Introducción}

La Asociación Española Universitaria de Traductores e Intérpretes en Formación (en adelante AETI) se fundó en el marco del IX Encuentro Nacional de Estudiantes de Traducción e Interpretación (ENETI), celebrado en Córdoba los días 13 y 14 de abril de 2012. En la asociación se fijan objetivos similares a los de cualquier asociación profesional, aunque se da una mayor importancia a la formación de los estudiantes y al vínculo entre la universidad, el mundo laboral y las asociaciones profesionales. Para cumplir con los objetivos especificados en los estatutos, la asociación ofrece a sus socios una variedad de recursos formativos y de comunicación entre socios, como son charlas, actividades y talleres organizados conjuntamente con las universidades o con las asociaciones profesionales, y una lista de distribución que imita el funcionamiento de las de otras asociaciones. Los socios deciden a qué nivel pueden implicarse en el trabajo de la asociación. Se puede participar libremente en comisiones, en las vocalías o representaciones de cada centro universitario o en la junta directiva.

El contacto entre socios, la cantidad de actividades organizadas desde la fundación de la asociación hasta el momento en que se hizo este estudio y la cercanía con las asociaciones profesionales nos llevó a reflexionar si los socios de AETI estaban expuestos a un input que influía en su desarrollo como futuros profesionales en este ámbito. Para ello se diseñó el estudio que a continuación presentamos, cuyo objetivo principal era identificar las competencias establecidas en el Libro blanco. Título de Grado en Traducción e Interpretación (ANECA, 2004) que los socios de $A E T I$ valoraban de manera superior que los estudiantes no socios.

El artículo está estructurado de la siguiente manera. En primer lugar se expone el marco conceptual del cual partimos. En segundo lugar exponemos los objetivos. En tercer lugar se exponen las hipótesis del estudio. En cuarto lugar describimos las variables. En quinto lugar se detalla la muestra del estudio. En sexto lugar se ofrece una explicación de la metodología usada. En séptimo lugar se presentan los resultados y en octavo lugar se formulan las conclusiones del estudio.

\section{La formación por competencias en los grados de Traducción e Interpretación}

\subsection{El papel de AETI en la formación de traductores e intérpretes}

AETI establece en sus estatutos dos grupos de objetivos principales. El primer grupo es el seguimiento de la formación en Traducción e Interpretación de las universidades españolas y el segundo es la promoción de actividades que ayuden a la formación de futuros traductores e intérpretes (estatutos de AETI, 2013). La asociación creó una serie de mecanismos de participación para que los socios se involucraran tanto en aquellos destinados a realizar el seguimiento de los estudios en Traducción e Interpretación - como la figura del vocal o la junta directivacomo en los que tenían por objetivo favorecer la formación de los socios en este ámbito -como los vocales y las comisiones-. Un año y medio después de la creación de la asociación, se había realizado un total de 28 actividades en 10 universidades: se organizó un concurso de traducción y uno de diseño, tres asambleas de vocales, se organizaron 19 charlas y actividades, se participó en dos congresos, se llevaron a cabo tres estudios de aspectos de la formación de los estudiantes de Traducción e Interpretación y se colaboró en la organización de dos seminarios. La participación en la lista de distribución reproducía las normas y el uso de las listas de las asociaciones profesionales, y el uso aumentaba cada mes.

\subsection{La formulación de competencias y selección para el estudio}

El Espacio Europeo de Educación Superior (EEES) se planteó como una reforma del sistema 
universitario europeo, tanto a nivel administrativo como a nivel pedagógico. La reforma pedagógica servía principalmente a dos objetivos. El primero era regular los estudios universitarios a escala europea para poder facilitar la equiparación de las titulaciones entre los países que se sumaran al EEES y así también favorecer la movilidad (Comunicado de Praga, 2001; Comunicado de Berlín, 2003). El segundo objetivo hacía referencia a adaptar la formación de las universidades europeas a la integración de un conocimiento explícito, funcional y aplicable a situaciones reales de manera efectiva (Comunicado de Berlín, 2003).

En España, los planes de estudio de los grados en Traducción e Interpretación se diseñaron a partir del Libro Blanco. Título de Grado en Traducción e Interpretación, publicado por la ANECA en 2004. En el libro se presenta un estudio, en el cual participaron las 18 universidades que en ese momento impartían la titulación completa de Traducción e Interpretación, que consistió en la obtención de información de agentes que tenían una actividad directa con la traducción e interpretación: egresados, empleadores y docentes. La muestra total aproximada fue de unos 523 sujetos. En el libro se determinan las competencias transversales genéricas (capítulo 6), las específicas (capítulo 7) y la asignación de las competencias a los perfiles profesionales (capítulo 8). En nuestro estudio partimos de las competencias establecidas en el Libro Blanco. Título de Grado en Traducción e Interpretación, porque este documento sirve de base para la formulación de las competencias de los planes de estudio de todas las universidades españolas y, por tanto, nos sirve para la evaluación de la valoración de todos los sujetos, independientemente de la universidad de donde procedan.

Para el estudio no partimos de todas las competencias que allí se establecen. Desde la asociación se realizó una primera selección de acuerdo con el tipo de tareas que los socios, los vocales, los participantes en las comisiones y la junta directiva realizaban. Las competencias seleccionadas fueron las siguientes:

- Competencias transversales instrumentales (CTI) (p. 81):

- CTI 1. Resolución de problemas

- CTI 2. Capacidad de análisis y síntesis

- CTI 4. Capacidad de gestión de la información

- CTI 5. Toma de decisiones

- Competencias transversales personales (CTP) (p. 82):

- CTP 1. Compromiso ético

- CTP 2. Habilidades en las relaciones interpersonales

- CTP 4. Trabajo en equipo

- Competencias transversales sistémicas (CTS) (p. 82-83):

- CTS 1. Motivación por la calidad

- CTS 2. Iniciativa y espíritu emprendedor

- CTS 3. Capacidad de aplicar los conocimientos a la práctica

- CTS 4. Capacidad de trabajo individual

- CTS 5. Diseño y gestión de proyectos

- Competencias específicas (CE) (p. 87-88):

- CE 1. Manejo de herramientas informáticas

- CE 2. Destreza para la búsqueda de información/documentación

- CE 3. Conocimiento de los aspectos económicos y profesionales

- CE 4. Capacidad de trabajo en equipo

- CE 5. Capacidad de diseñar y gestionar proyectos

A partir de estas competencias se formularon las variables dependientes del estudio.

\section{Objetivos}

Los objetivos del presente artículo son: 1) identificar las competencias que los socios de AETI desarrollan a un nivel mayor que los estudiantes no socios y 2) determinar si existe una 
relación positiva entre la valoración de las competencias identificadas y la implicación en el trabajo de la asociación y la antigüedad de pertenencia.

\section{Hipótesis}

Exponemos a continuación las hipótesis que formulamos para nuestro estudio.

\subsection{Hipótesis general}

La pertenencia e implicación en la Asociación Española Universitaria de Estudiantes de Traducción e Interpretación ayuda en el proceso de adquisición y desarrollo de ciertas competencias de la formación de traductores e intérpretes en España.

\subsection{Primera hipótesis de estudio}

En las competencias identificadas con un ascenso de la valoración de los socios de AETI respecto a los no socios, la valoración que los sujetos hacen sobre las competencias depende de su nivel de implicación en la asociación.

\subsection{Segunda hipótesis de estudio}

En las competencias identificadas con un ascenso de la valoración de los socios de AETI respecto a los no socios, la valoración que los sujetos hacen sobre las competencias depende del grado de antigüedad del sujeto en la asociación.

\section{Variables}

Exponemos a continuación las variables dependientes e independientes del estudio.

\subsection{Independientes}

En nuestro estudio trabajamos con tres variables independientes, categorizadas de la siguiente manera:

- Pertenencia a la asociación: distinguimos entre los sujetos que pertenecen a AETI y los que no.
- Nivel de implicación: distinguimos entre los siguientes niveles de implicación en AETI:

-implicación o: sujetos que no han sido miembros de AETI;

_implicación 1: socios que no participan o no han participado en comisiones, vocalías o junta directiva;

-implicación 2: socios que participan o han participado en comisiones o vocalías;

-implicación 3: socios que participan o han participado en la junta directiva.

- Antigüedad: distinguimos entre los siguientes grados de antigüedad de pertenencia a la asociación:

- antigüedad o: sujetos que no han sido miembros de AETI;

— antigüedad 1 : socios que se inscribieron a AETI entre los meses de febrero a mayo de 2013;

- antigüedad 2: socios que se inscribieron a AETI entre los meses de noviembre de 2012 a enero de 2013;

- antigüedad 3: socios que se inscribieron a AETI entre los meses de agosto a octubre de 2012;

—antigüedad 4: socios que se inscribieron a AETI entre los meses de mayo a julio de 2012.

\subsection{Dependientes}

Las variables dependientes que usamos en nuestro estudio son las valoraciones de las competencias que enumerábamos en el punto 2.

\section{Universo y muestra}

El universo de estudio son los estudiantes de grado y de licenciatura en Traducción e Interpretación que son socios de AETI y los estudiantes de grado y de licenciatura en Traducción e Interpretación que no son socios de AETI.

La muestra está compuesta por 120 sujetos: 60 estudiantes socios de AETI y 60 estudiantes que no son socios. En cada grupo muestral de 60 sujetos hay 20 por cada ciclo. 
Para comprobar que los grupos muestrales estuvieran formados homogéneamente y evitar que las variables extrañas tuvieran un efecto excesivamente grande en las mediciones, se hizo una primera criba de los sujetos. Se eliminaron todos los que habían indicado que pertenecían a otra asociación, ya que podían no ser socios de AETI y adquirir las competencias que queríamos medir en otras asociaciones. Se eliminaron los sujetos miembros de AETI que se habían inscrito en los meses de junio, julio y agosto de 2013, porque la encuesta se realizó en agosto de 2013 y a partir de junio la actividad de la asociación disminuye a causa del fin del curso académico.

Nos importaba saber si el tipo de estudio que se había cursado actuaba como variable independiente. Por ello, se comprobó si las variables seguían la distribución uniforme discreta para así aplicar un test de comparación de grupos con contraste bilateral adecuado. En el apéndice 2 se encuentran los resultados del test de bondad de ajuste. Al no seguir ninguna variable la distribución uniforme discreta, se aplicó el test de Mann-Whitney. Solamente dos variables presentan diferencias estadísticamente significativas (variable 12: estadístico U: 1197.5; estadístico Z: -2.652; p-valor: 0.008; variable 15: estadístico U: 1125; estadístico Z: -3.066; p-valor: 0.002) y, por tanto, al tratarse de una minoría, concluimos que el tipo de estudio cursado no actúa como variable independiente.

El siguiente paso fue organizar los cursos por ciclos. Distinguimos entre tres ciclos de formación de traductores: un primer ciclo compuesto por el primer y segundo curso, cuyo objetivo es adquirir los conocimientos y competencias esenciales para la traducción general; un segundo ciclo compuesto por tercer, cuarto y quinto curso en el caso de las licenciaturas de cinco años, cuyo objetivo es consolidar los conocimientos y competencias esenciales para la traducción general e iniciar las especializaciones, y un tercer ciclo compuesto por egresados y estudiantes de posgrado, en el cual los sujetos han finalizado o amplían su formación universitaria como traductores e intérpretes.

Mediante esta reorganización, obtuvimos el recuento de los sujetos de cada grupo muestral que se presenta en el siguiente cuadro. Para trabajar con grupos del mismo tamaño, se decidió reducir el total de sujetos por grupo a 20:

\section{TABLA 1}

Distribución de la muestra inicial y selección al azar

\begin{tabular}{cccccc} 
SOCIO & $\mathbf{1}^{\text {er }}$ CICLO & $\mathbf{2}^{\mathbf{0}}$ CICLO & $\mathbf{3}^{\text {er }}$ CICLO & TOTAL \\
\cline { 2 - 6 } Sí & 27 & 32 & 23 & 82 \\
\cline { 2 - 6 } & -7 & -12 & -3 & -22 \\
\cline { 2 - 5 } & 20 & 20 & 20 & 60 \\
\hline \multirow{3}{*}{ NO } & 38 & 44 & 26 & 108 \\
\cline { 2 - 6 } & -18 & -24 & -6 & -48 \\
\cline { 2 - 6 } & 20 & 20 & 20 & 60 \\
\hline
\end{tabular}

Para garantizar la aleatoriedad de la muestra, se usó un procedimiento de eliminación basado en el azar. Una vez que todos los grupos estuvieron formados por veinte sujetos, se realizó la prueba de rachas para comprobar la aleatoriedad de la muestra. Los resultados obtenidos se presentan en la tabla 2.

Podemos concluir que es aleatoria. La muestra final utilizada se compone de 120 sujetos: 60 que son socios de AETI y 60 que no lo son. De los 120 sujetos, 44 son estudiantes de licenciatura y 76 de grado. De los 60 socios de AETI, 44 se sitúan en un nivel de implicación 1 , 9 en un nivel de implicación 2 y 7 en un nivel de implicación 3. De los 60 socios de AETI, 6 socios tienen una antigüedad de pertenencia a la asociación de 4 a 7 meses, 14 socios de 8 a 10 meses, 11 socios de 11 a 13 meses y 29 socios de 14 a 16 meses.

\section{Metodología}

\subsection{Diseño de la encuesta}

Como instrumento de recogida de datos, se diseñó una encuesta en línea. Para el diseño del 


\section{TABLA 2}

Resultados del test de rachas para cada variable

\begin{tabular}{|c|c|c|c|c|}
\hline & Hipótesis nula & Test & Sig. & Decisión \\
\hline 1 & $\begin{array}{l}\text { La secuencia de valores definida por } \\
\text { V_1<=10,00 y }>10,00 \text { es aleatoria. }\end{array}$ & $\begin{array}{l}\text { Prueba de ejecuciones } \\
\text { de una muestra }\end{array}$ & ,600 & $\begin{array}{l}\text { Retener la } \\
\text { hipótesis nula. }\end{array}$ \\
\hline 2 & $\begin{array}{l}\text { La secuencia de valores definida por } \\
\vee \_2<=11,00 \text { y }>11,00 \text { es aleatoria. }\end{array}$ & $\begin{array}{l}\text { Prueba de ejecuciones } \\
\text { de una muestra }\end{array}$ & 801 & $\begin{array}{l}\text { Retener la } \\
\text { hipótesis nula. }\end{array}$ \\
\hline 3 & $\begin{array}{l}\text { La secuencia de valores definida por } \\
\vee \_3<=9,00 \text { y }>9,00 \text { es aleatoria. }\end{array}$ & $\begin{array}{l}\text { Prueba de ejecuciones } \\
\text { de una muestra }\end{array}$ & 330 & $\begin{array}{l}\text { Retener la } \\
\text { hipótesis nula. }\end{array}$ \\
\hline 4 & $\begin{array}{l}\text { La secuencia de valores definida por } \\
\text { V_4<=10,00 y }>10,00 \text { es aleatoria. }\end{array}$ & $\begin{array}{l}\text { Prueba de ejecuciones } \\
\text { de una muestra }\end{array}$ & 845 & $\begin{array}{l}\text { Retener la } \\
\text { hipótesis nula. }\end{array}$ \\
\hline 5 & $\begin{array}{l}\text { La secuencia de valores definida por } \\
\text { V_5 }<=10,00 \text { y }>10,00 \text { es aleatoria. }\end{array}$ & $\begin{array}{l}\text { Prueba de ejecuciones } \\
\text { de una muestra }\end{array}$ &, 545 & $\begin{array}{l}\text { Retener la } \\
\text { hipótesis nula. }\end{array}$ \\
\hline 6 & $\begin{array}{l}\text { La secuencia de valores definida por } \\
\vee \_6<=10,00 \text { y }>10,00 \text { es aleatoria. }\end{array}$ & $\begin{array}{l}\text { Prueba de ejecuciones } \\
\text { de una muestra }\end{array}$ & ,684 & $\begin{array}{l}\text { Retener la } \\
\text { hipótesis nula. }\end{array}$ \\
\hline 7 & $\begin{array}{l}\text { La secuencia de valores definida por } \\
\vee \_7<=11,00 \text { y }>11,00 \text { es aleatoria. }\end{array}$ & $\begin{array}{l}\text { Prueba de ejecuciones } \\
\text { de una muestra }\end{array}$ & 071 & $\begin{array}{l}\text { Retener la } \\
\text { hipótesis nula. }\end{array}$ \\
\hline 8 & $\begin{array}{l}\text { La secuencia de valores definida por } \\
\text { v_ } 8<=9,00 \text { y }>9,00 \text { es aleatoria. }\end{array}$ & $\begin{array}{l}\text { Prueba de ejecuciones } \\
\text { de una muestra }\end{array}$ &, 278 & $\begin{array}{l}\text { Retener la } \\
\text { hipótesis nula. }\end{array}$ \\
\hline 9 & $\begin{array}{l}\text { La secuencia de valores definida por } \\
\text { ﹎_ } 9<=7,00 \text { y }>7,00 \text { es aleatoria. }\end{array}$ & $\begin{array}{l}\text { Prueba de ejecuciones } \\
\text { de una muestra }\end{array}$ & 841 & $\begin{array}{l}\text { Retener la } \\
\text { hipótesis nula. }\end{array}$ \\
\hline 10 & $\begin{array}{l}\text { La secuencia de valores definida por } \\
\vee \_10<=11,00 \text { y }>11,00 \text { es aleatoria. }\end{array}$ & $\begin{array}{l}\text { Prueba de ejecuciones } \\
\text { de una muestra }\end{array}$ & 964 & $\begin{array}{l}\text { Retener la } \\
\text { hipótesis nula. }\end{array}$ \\
\hline 11 & $\begin{array}{l}\text { La secuencia de valores definida por } \\
\vee \_11<=10,00 \text { y }>10,00 \text { es aleatoria. }\end{array}$ & $\begin{array}{l}\text { Prueba de ejecuciones } \\
\text { de una muestra }\end{array}$ & ,600 & $\begin{array}{l}\text { Retener la } \\
\text { hipótesis nula. }\end{array}$ \\
\hline 12 & $\begin{array}{l}\text { La secuencia de valores definida por } \\
v_{-} 12<=8,00 \text { y }>8,00 \text { es aleatoria. }\end{array}$ & $\begin{array}{l}\text { Prueba de ejecuciones } \\
\text { de una muestra }\end{array}$ & 312 & $\begin{array}{l}\text { Retener la } \\
\text { hipótesis nula. }\end{array}$ \\
\hline 13 & $\begin{array}{l}\text { La secuencia de valores definida por } \\
\text { V_13<=10,00 y }>10,00 \text { es aleatoria. }\end{array}$ & $\begin{array}{l}\text { Prueba de ejecuciones } \\
\text { de una muestra }\end{array}$ & 600 & $\begin{array}{l}\text { Retener la } \\
\text { hipótesis nula. }\end{array}$ \\
\hline 14 & $\begin{array}{l}\text { La secuencia de valores definida por } \\
\text { V_14<=9,00 y }>9,00 \text { es aleatoria. }\end{array}$ & $\begin{array}{l}\text { Prueba de ejecuciones } \\
\text { de una muestra }\end{array}$ &, 353 & $\begin{array}{l}\text { Retener la } \\
\text { hipótesis nula. }\end{array}$ \\
\hline 15 & $\begin{array}{l}\text { La secuencia de valores definida por } \\
\vee \_15<=10,00 \text { y }>10,00 \text { es aleatoria. }\end{array}$ & $\begin{array}{l}\text { Prueba de ejecuciones } \\
\text { de una muestra }\end{array}$ & ,493 & $\begin{array}{l}\text { Retener la } \\
\text { hipótesis nula. }\end{array}$ \\
\hline
\end{tabular}

Se muestran las significancias asintóticas. El nivel de significancia es,05.

instrumento se siguieron las siguientes fases: 1) formulación de los ítems, 2) prueba piloto de la encuesta y 3) análisis de los resultados de la prueba piloto e introducción de las modificaciones pertinentes. A continuación las comentamos brevemente.

\subsubsection{Formulación de los ítems}

Se decidió utilizar una adaptación de las escalas de Likert en termómetros de opinión de seis valores para los ítems. Para cada competencia se formularon tres ítems. En la mayoría de las competencias, de los tres ítems uno se formuló con una redacción negativa. Esta decisión se tomó para favorecer la concentración del sujeto a la hora de responder los ítems. Se mezclaron todos los ítems entre sí para disminuir la repetitividad de la encuesta. El resultado fue una encuesta de 45 ítems para 15 competencias.

\subsubsection{Prueba piloto}

Se obtuvieron diez respuestas del grupo muestral de socios de AETI con nivel de implicación 3 (miembros de la junta directiva). Se midió 
la desviación estándar de las respuestas y el índice de confiabilidad $\alpha$ de Cronbach de las competencias.

\subsubsection{Análisis de los resultados de la prueba piloto}

La mayor desviación estándar registrada fue de 1.4719. En cuanto al índice de confiabilidad de la encuesta, de las 15 competencias solamente cuatro tenían un índice igual o mayor a 0.800. Una vez observados los valores del $\alpha$ de Cronbach para ver si se eliminaba algún elemento de la escala, se decidió que estas solamente estuvieran formadas por dos ítems. Mediante esta decisión, se aumentó la cantidad de competencias con un índice aceptable de cuatro a siete. En los casos en los que aún no se superaba el índice mínimo de 0.800 , se revisó la formulación de uno de los dos ítems (el que presentaba una desviación estándar mayor).

Una vez eliminados o reformulados los ítems, se calculó el $\alpha$ de Cronbach general de la encuesta. El resultado fue de 0.895 , superior al nivel mínimo de confiabilidad. En el apéndice 3 se encuentran los datos relacionados con el $\alpha$ de cronbach.

Se corrigió la encuesta y los ítems se desordenaron de nuevo, obteniendo así una encuesta de 30 ítems. Puede encontrarse una copia de la encuesta en el apéndice 1.

\subsection{Estadística}

Para contrastar las hipótesis planteadas, usamos técnicas de relación entre variables y de comparación entre grupos. Los cálculos estadísticos se han realizado con el programa SPSS V.20.

Se utiliza el test de bondad de ajuste de Komogórov-Smirnov para determinar si las variables siguen la distribución uniforme discreta. En el apéndice 2 se encuentran los resultados según los grupos muestrales. Para comprobar las hipótesis operacionales de las hipótesis de estudio 1 y 2, utilizamos la técnica estadística de relación de la correlación de Spearman. En el caso de la comparación de los grupos, se utiliza el test de Mann-Whitney.

En todos los test se usa un nivel de significación unilateral de 0.025. El nivel de significación se ofrece mediante el estadístico p-valor.

\section{Resultados}

Los resultados se ofrecen por variable dependiente y se estructuran de la siguiente manera: 1) se dan las medias por variable independiente de pertenencia a la asociación, 2) se ofrecen los resultados de los test de comparación entre los dos grupos, 3) se aportan los resultados del test de relación entre la valoración de la variable y el nivel de implicación en la asociación y los resultados de la relación entre la valoración de la variable y el grado de antigüedad de pertenencia a la asociación. Las medias van acompañadas del valor de \pm 2 errores estándares. Para la interpretación de las medias, se utiliza la siguiente categorización:

\section{TABLA 3}

Categorización e interpretación de las escalas

\begin{tabular}{cc} 
Valor & Interpretación \\
\hline $10-12$ & Valoración muy alta \\
\hline $8-9.99$ & Valoración alta \\
\hline $6-7.99$ & Valoración media \\
\hline $4-5.99$ & Valoración baja \\
\hline $2-3.99$ & Valoración muy baja \\
\hline
\end{tabular}

\subsection{Valoración de la capacidad de apli- car los conocimientos a la práctica}

Los sujetos que son miembros de AETI valoran su capacidad de aplicar los conocimientos a la práctica en un 10.12ะ0.395 sobre 12 (valoración muy alta). En el caso de los sujetos que no son socios, la valoración es de un 10.03ะ0.436 sobre 12 (valoración muy alta). 
No hay un ascenso estadísticamente significativo de la valoración de los sujetos que son socios respecto a los sujetos que no lo son (estadístico U: 1792.5; estadístico Z: -0.04; p-valor: 0.484).

No hay una relación positiva estadísticamente significativa entre la valoración de la variable y el nivel de implicación en la asociación (correlación de Spearman: -0.002; p-valor: 0.491). Tampoco hay una relación positiva estadísticamente significativa entre la valoración de la variable y el grado de antigüedad de pertenencia a la asociación (correlación de Spearman: 0.64; p-valor: 0.242).

\subsection{Valoración del conocimiento de los aspectos económicos y profesionales}

Los sujetos que son miembros de AETI valoran su conocimiento de los aspectos económicos y profesionales en un $10.55 \pm 0.306$ sobre 12 (valoración muy alta). En el caso de los sujetos que no son socios, la valoración es de un 9.85×0.392 sobre 12 (valoración alta).

Hay un ascenso estadísticamente significativo de la valoración de los sujetos que son socios respecto a los sujetos que no lo son (estadístico U: 1316; estadístico Z: -2.607; p-valor: 0.005).

Hay una relación positiva estadísticamente significativa entre la valoración de la variable y el nivel de implicación en la asociación (correlación de Spearman: 0.241; p-valor: 0.004). También hay una relación positiva estadísticamente significativa entre la valoración de la variable y el grado de antigüedad de pertenencia a la asociación (correlación de Spearman: 0.266; p-valor: 0.002).

\subsection{Valoración de la capacidad de ges- tión de la información}

Los sujetos que son miembros de AETI valoran su capacidad de gestión de la información en un 8.82 0.437 sobre 12 (valoración alta). En el caso de los sujetos que no son socios, la valoración es de un 9.05ะ0.416 sobre 12 (valoración alta).
No hay un ascenso estadísticamente significativo de la valoración de los sujetos que son socios respecto a los sujetos que no lo son (estadístico U: 1681; estadístico Z: -0.636; p-valor: 0.263).

No hay una relación positiva estadísticamente significativa entre la valoración de la variable y el nivel de implicación en la asociación (correlación de Spearman: -0.097; p-valor: 0.145). Tampoco hay una relación positiva estadísticamente significativa entre la valoración de la variable y el grado de antigüedad de pertenencia a la asociación (correlación de Spearman: -0.030; p-valor: 0.371).

\subsection{Valoración del manejo de herra- mientas informáticas}

Los sujetos que son miembros de AETI valoran su manejo de herramientas informáticas en un 9.83 \pm 0.471 sobre 12 (valoración alta). En el caso de los sujetos que no son socios, la valoración es de un 9.27士0.503 sobre 12 (valoración alta).

No hay un ascenso estadísticamente significativo de la valoración de los sujetos que son socios respecto a los sujetos que no lo son (estadístico U: 1462; estadístico Z: -1.801; p-valor: 0.036).

No hay una relación positiva estadísticamente significativa entre la valoración de la variable y el nivel de implicación en la asociación (correlación de Spearman: 0.174; p-valor: 0.029). Sin embargo, sí hay una relación positiva estadísticamente significativa entre la valoración de la variable y el grado de antigüedad de pertenencia a la asociación (correlación de Spearman: 0.184; p-valor: 0.022).

\subsection{Valoración de la destreza para la búsqueda de información y de docu- mentación}

Los sujetos que son miembros de AETI vaIoran su destreza para la búsqueda de información y de documentación en un 9.37£0.371 sobre 12 (valoración alta). En el caso de los sujetos que 
no son socios, la valoración es de un 8.57ะ0.402 sobre 12 (valoración alta).

Hay un ascenso estadísticamente significativo de la valoración de los sujetos que son socios respecto a los sujetos que no lo son (estadístico U: 1264; estadístico Z: -2.873; p-valor: 0.002).

Hay una relación positiva estadísticamente significativa entre la valoración de la variable y el nivel de implicación en la asociación (correlación de Spearman: 0.224; p-valor: 0.007). También hay una relación positiva estadísticamente significativa entre la valoración de la variable y el grado de antigüedad de pertenencia a la asociación (correlación de Spearman: 0.280; p-valor: $0.001)$.

\subsection{Valoración de la capacidad de análi- sis y de síntesis}

Los sujetos que son miembros de AETI valoran su capacidad de análisis y de síntesis en un $9.50 \pm 0.338$ sobre 12 (valoración alta). En el caso de los sujetos que no son socios, la valoración es de un 9.47ะ0.385 sobre 12 (valoración alta).

No hay un ascenso estadísticamente significativo de la valoración de los sujetos que son socios respecto a los sujetos que no lo son (estadístico U: 1746; estadístico Z: -0.29; p-valor: 0.386).

No hay una relación positiva estadísticamente significativa entre la valoración de la variable y el nivel de implicación en la asociación (correlación de Spearman: 0.004; p-valor: 0.484). Tampoco hay una relación positiva estadísticamente significativa entre la valoración de la variable y el grado de antigüedad de pertenencia a la asociación (correlación de Spearman: 0.71; p-valor: 0.220).

\subsection{Valoración de las habilidades en las relaciones interpersonales}

Los sujetos que son miembros de AETI valoran sus habilidades en las relaciones interpersonales en un $11 \pm 0.223$ sobre 12 (valoración muy alta). En el caso de los sujetos que no son socios, la valoración es de un 10.32ะ0.426 sobre 12 (valoración muy alta).

Hay un ascenso estadísticamente significativo de la valoración de los sujetos que son socios respecto a los sujetos que no lo son (estadístico U: 1427; estadístico Z: -2.039; p-valor: 0.021).

Hay una relación positiva estadísticamente significativa entre la valoración de la variable y el nivel de implicación en la asociación (correlación de Spearman: 0.178; p-valor: 0.024). También hay una relación positiva estadísticamente significativa entre la valoración de la variable y el grado de antigüedad de pertenencia a la asociación (correlación de Spearman: 0.219; p-valor: 0.008)

\subsection{Valoración de la capacidad de traba- jo en equipo}

Los sujetos que son miembros de AETI vaIoran su capacidad de trabajo en equipo en un $9.20 \pm 0.361$ sobre 12 (valoración alta). En el caso de los sujetos que no son socios, la valoración es de un 8.48ะ0.524 sobre 12 (valoración alta).

Hay un ascenso estadísticamente significativo de la valoración de los sujetos que son socios respecto a los sujetos que no lo son (estadístico U: 1410; estadístico Z: -2.083; p-valor: 0.019).

Hay una relación positiva estadísticamente significativa entre la valoración de la variable y el nivel de implicación en la asociación (correlación de Spearman: 0.188; p-valor: 0.020). Sin embargo, no hay una relación positiva estadísticamente significativa entre la valoración de la variable y el grado de antigüedad de pertenencia a la asociación (correlación de Spearman: 0.164; p-valor: 0.037).

\subsection{Valoración de la capacidad de traba- jo individual}

Los sujetos que son miembros de AETI vaIoran su capacidad de trabajo individual en un 
7.37ะ0.496 sobre 12 (valoración media). En el caso de los sujetos que no son socios, la valoración es de un 7.45×0.571 sobre 12 (valoración media).

No hay un ascenso estadísticamente significativo de la valoración de los sujetos que son socios respecto a los sujetos que no lo son (estadístico U: 1796; estadístico Z: -0.021; p-valor: 0.492).

No hay una relación positiva estadísticamente significativa entre la valoración de la variable y el nivel de implicación en la asociación (correlación de Spearman: -0.060; p-valor: 0.258). Tampoco no hay una relación positiva estadísticamente significativa entre la valoración de la variable y el grado de antigüedad de pertenencia a la asociación (correlación de Spearman: 0.012; p-valor: 0.449).

\subsection{Valoración de la motivación por la calidad}

Los sujetos que son miembros de AETI valoran su motivación por la calidad en un 10.58 0.378 sobre 12 (valoración muy alta). En el caso de los sujetos que no son socios, la valoración es de un 10.52ะ0.364 sobre 12 (valoración muy alta).

No hay un ascenso estadísticamente significativo de la valoración de los sujetos que son socios respecto a los sujetos que no lo son (estadístico U: 1739.5; estadístico Z: -0.328; p-valor: 0.372).

No hay una relación positiva estadísticamente significativa entre la valoración de la variable y el nivel de implicación en la asociación (correlación de Spearman: 0.000; p-valor: 0.498). Tampoco hay una relación positiva estadísticamente significativa entre la valoración de la variable y el grado de antigüedad de pertenencia a la asociación (correlación de Spearman: 0.028; p-valor: 0.380).

\subsection{Valoración de la iniciativa y el espí- ritu emprendedor}

Los sujetos que son miembros de AETI valoran su iniciativa y su espíritu emprendedor en un
10.30£0.323 sobre 12 (valoración muy alta). En el caso de los sujetos que no son socios, la valoración es de un 9.60ะ0.387 sobre 12 (valoración alta).

Hay un ascenso estadísticamente significativo de la valoración de los sujetos que son socios respecto a los sujetos que no lo son (estadístico U: 1313.5; estadístico Z: -2.612; p-valor: 0.005).

Hay una relación positiva estadísticamente significativa entre la valoración de la variable y el nivel de implicación en la asociación (correlación de Spearman: 0.251; p-valor: 0.003). También hay una relación positiva estadísticamente significativa entre la valoración de la variable y el grado de antigüedad de pertenencia a la asociación (correlación de Spearman: 0.266; p-valor: 0.002).

\subsection{Valoración de la capacidad de dise- ñar y gestionar proyectos}

Los sujetos que son miembros de AETI valoran su capacidad de diseñar y gestionar proyectos en un $7.63 \pm 0.336$ sobre 12 (valoración media). En el caso de los sujetos que no son socios, la valoración es de un 7.02ะ0.334 sobre 12 (valoración media).

Hay un ascenso estadísticamente significativo de la valoración de los sujetos que son socios respecto a los sujetos que no lo son (estadístico U: 1328.5; estadístico Z: -2.54; p-valor: 0.006).

Hay una relación positiva estadísticamente significativa entre la valoración de la variable y el nivel de implicación en la asociación (correlación de Spearman: 0.222; p-valor: 0.007). También hay una relación positiva estadísticamente significativa entre la valoración de la variable y el grado de antigüedad de pertenencia a la asociación (correlación de Spearman: 0.232; p-valor: 0.005).

\subsection{Valoración del compromiso ético}

Los sujetos que son miembros de AETI valoran su compromiso ético en un 9.78ะ0.401 sobre 
12 (valoración alta). En el caso de los sujetos que no son socios, la valoración es de un 9.90ะ0.414 sobre 12 (valoración alta).

No hay un ascenso estadísticamente significativo de la valoración de los sujetos que son socios respecto a los sujetos que no lo son (estadístico U: 1698.5; estadístico Z: -0.543; p-valor: 0.294).

No hay una relación positiva estadísticamente significativa entre la valoración de la variable y el nivel de implicación en la asociación (correlación de Spearman: -0.018; p-valor: 0.423). Tampoco hay una relación positiva estadísticamente significativa entre la valoración de la variable y el grado de antigüedad de pertenencia a la asociación (-0.041; p-valor: 0.328).

\subsection{Valoración de la resolución de pro- blemas}

Los sujetos que son miembros de AETI valoran su capacidad de resolución de problemas en un $10 \pm 0.353$ sobre 12 (valoración muy alta). En el caso de los sujetos que no son socios, la valoración es de un 9.40ะ0.378 sobre 12 (valoración alta).

Hay un ascenso estadísticamente significativo de la valoración de los sujetos que son socios respecto a los sujetos que no lo son (estadístico U: 1352; estadístico Z: -2.404; p-valor: 0.008).

Hay una relación positiva estadísticamente significativa entre la valoración de la variable y el nivel de implicación en la asociación (correlación de Spearman: 0.207; p-valor: 0.012). También hay una relación positiva estadísticamente significativa entre la valoración de la variable y el grado de antigüedad de pertenencia a la asociación (correlación de Spearman: 0.207; p-valor: 0.012).

\subsection{Valoración de la toma de decisio- nes}

Los sujetos que son miembros de AETI valoran su capacidad de toma de decisiones en un
9.95 \pm 0.320 sobre 12 (valoración alta). En el caso de los sujetos que no son socios, la valoración es de un 10.05£0.363 sobre 12 (valoración muy alta).

No hay un ascenso estadísticamente significativo de la valoración de los sujetos que son socios respecto a los sujetos que no lo son (estadístico U: 1680.5; estadístico Z: -0.646; p-valor: 0.260).

No hay una relación positiva estadísticamente significativa entre la valoración de la variable y el nivel de implicación en la asociación (correlación de Spearman: -0.052; p-valor: 0.287). No hay una relación positiva estadísticamente significativa entre la valoración de la variable y el grado de antigüedad de pertenencia a la asociación (correlación de Spearman: -0.025; p-valor: 0.394).

\section{Conclusiones}

A continuación presentamos las conclusiones más relevantes del estudio.

\subsection{Identificación de las competencias que los socios de AETI desarrollan a un nivel mayor que los estudiantes no so- cios}

De las quince variables dependientes planteadas, hemos obtenido ascensos estadísticamente significativos en siete: 1) el conocimiento de aspectos económicos y profesionales, 2) la destreza para la búsqueda de información y documentación, 3) las habilidades en las relaciones interpersonales, 4) la capacidad de trabajo en grupo, 5) la iniciativa y el espíritu emprendedor, 6) la capacidad de diseñar y gestionar proyectos y 7) la capacidad de resolución de problemas.

Mediante este estudio hemos podido identificar qué competencias se desarrollan cuando se es socio de AETI. Esta información nos ayuda a diseñar actividades y proyectos que potencien la adquisición y desarrollo de estas competencias y también que incluyan aquellas en las cuales no se han encontrado diferencias. 


\subsection{Primera y segunda hipótesis de es- tudio}

En nuestra segunda hipótesis de estudio afirmábamos que la valoración de las competencias está relacionada con el nivel de implicación en la asociación y en la tercera que la valoración de las competencias está relacionada con el grado de antigüedad de pertenencia a la asociación. De las treinta variables dependientes planteadas, hemos obtenido dieciséis relaciones positivas estadísticamente significativas, aunque la fuerza de relación es baja. Tanto en la implicación como en la antigüedad se observan relaciones positivas estadísticamente significativas en: 1) el conocimiento de aspectos económicos y profesionales, 2) la destreza para la búsqueda de información y documentación, 3) las habilidades en las relaciones interpersonales, 4) la iniciativa y el espíritu emprendedor, 5) la capacidad de diseñar y gestionar proyectos y 6) la capacidad de resolución de problemas. Además, en la implicación también se observa la capacidad de trabajo en grupo y en la antigüedad el manejo de herramientas informáticas.

En general, en las variables en las cuales se observa un ascenso estadísticamente significativo de la valoración de las competencias, podemos afirmar que existe una relación positiva entre la valoración de las competencias, el nivel de implicación en la asociación y el grado de antigüedad de pertenencia a la asociación, aunque en general es una relación de baja intensidad. Con esto podemos confirmar que la tendencia es que cuanto mayor es el provecho de los recursos, los proyectos y las actividades de la asociación y el trabajo para la organización, mayor es el nivel de adquisición y desarrollo de las competencias en las cuales hemos encontrado un ascenso estadísticamente significativo en la valoración de los socios respecto a los que no lo son. También podemos confirmar que cuanto más tiempo llevan los socios implicados en la asociación, mayor es su valoración de las competencias. Estos factores demuestran indirectamente lo que ya apuntábamos en el punto "La formación por competencias en el grado de Traducción e Interpretación".

Cuando hablamos de que la pertenencia a la asociación ayuda en la adquisición de competencias, no nos referimos al hecho de inscribirse, pagar una cuota y desentenderse de todo el proyecto de AETI. El uso de los recursos e instrumentos de la asociación, la participación en las actividades, la participación en los órganos de organización de la asociación y, en general, tener un papel activo en cada una de las categorías de implicación que hemos establecido es lo que en realidad está ayudando al miembro a adquirir y desarrollar sus competencias.

\subsection{Hipótesis general}

Nuestra hipótesis general es que la pertenencia y la implicación en la Asociación Española Universitaria de Estudiantes de Traducción e Interpretación ayudan en el proceso de adquisición y desarrollo de ciertas competencias de la formación de traductores e intérpretes en España. Con los resultados obtenidos, hemos podido constatar que la pertenencia y la implicación en la asociación ayudan en la adquisición de ciertas competencias.

Si bien es cierto que no hemos podido corroborar que se favorece la adquisición y desarrollo de las quince que habíamos seleccionado en un primer momento, las siete competencias que presentan una valoración más alta en el grupo compuesto por socios nos parecen esenciales para la formación de traductores e intérpretes, sobre todo para su inserción en el mundo laboral. La capacidad de trabajar en equipo y las habilidades en las relaciones interpersonales son competencias que necesitarán poseer en cualquier perfil profesional relacionado con el mundo de la traducción y de la interpretación, así como en la socialización con otros profesionales en un entorno de colaboración como el que se da 
en las redes sociales o en las asociaciones profesionales. La iniciativa y el espíritu emprendedor, la capacidad de diseñar y gestionar proyectos y la capacidad de resolución de problemas son también competencias clave en la formulación de un proyecto formativo y profesional propio. Finalmente, una buena destreza para la búsqueda de información y de documentación es la mejor aliada de todo traductor e intérprete.

La pertenencia y la implicación en la Asociación Española Universitaria de Traductores e Intérpretes en Formación supone un buen complemento a la formación universitaria, porque favorece 1) la adquisición y desarrollo de ciertas competencias establecidas en el Libro blanco. Título de Grado en Traducción e Interpretación (ANECA, 2004), 2) la socialización del estudiante mediante las herramientas de comunicación de la asociación y 3) el acercamiento al mundo profesional mediante las actividades que se desarrollan juntamente con las asociaciones profesionales.

\section{Bibliografía citada}

Agencia Nacional de Evaluación de la Calidad y AcrediTACION, 2004: Libro blanco. Título de Grado en Traducción e Interpretación, España: ANECA. [http:// www.aneca.es/var/media/150288/libroblanco_ traduc_def.pdf, fecha de consulta: 30/7/2014].

Alvira Martín, Francisco, 2004: La Encuesta: una perspectiva general metodológica, Madrid: Centro de Investigaciones Sociológicas.

Asociación Española Universitaria de Traductores e Intérpretes en Formación, 2013: Estatutos de la AETI, España: Asociación Española Universitaria de Traductores e Intérpretes en Formación [http:// aetioficial.es/quienes-somos/estatutos/, fecha de consulta: 30/7/2014].

Declaración de Praga, 2001: Hacia el Área de la Educación Superior Europea. Declaración del encuentro de los Ministros Europeos en funciones de la Educación Superior en Praga, 19 de mayo del 2001 [http://www.eees.es/pdf/Praga_ES.pdf, fecha de consulta: 30/7/2014]

Declaración de Berlín, 2003: Educación Superior Europea. Comunicado de la Conferencia de Ministros responsables de la Educación Superior, mantenida en Berlín el 19 de Septiembre de 2003 [http://www.eees.es/pdf/Berlin_ES.pdf, fecha de consulta: 30/7/2014].

Hernandez Sampieri, Roberto, 2006: Metodología de la investigación (4a ed.), México D.F. [etc.]: McGraw-Hill.

Losada López, José Luis y Rafael López-FeAL, 2002: Métodos de investigación en ciencias humanas y sociales, Madrid: Thomson.

Neunzig, Wilhelm y Helena Tanqueiro, 2007: Estudios empíricos en traducción: enfoques y métodos, [Bellaterra] Girona: Universitat Autònoma de Barcelona. Departament de Traducció i d'Interpretació; Documenta Universitaria.

Santisteban Requena, Carmen, 2009: Principios de psicometría, Madrid: Síntesis.

Pardo Merino, Antonio y Rafael San Martín CastellaNos, 2010. Análisis de datos en ciencias sociales y de la salud II, Madrid: Síntesis.

\section{Apéndices}

1) Encuesta: En el siguiente enlace se ofrece una reproducción íntegra de la encuesta utilizada en línea:

http://bit.ly/encuesta_competencias_AETI.

2) Test de bondad de ajuste: en el siguiente enlace se ofrecen los resultados de los test de bondad de ajuste de Kolmogórov-Smirnov por grupo muestral:

http://bit.ly/distribucion_uniforme.

3) A de Cronbach: en el siguiente enlace se ofrecen los resultados del $\alpha$ de Cronbach de los ítems y las decisiones posteriores: http://bit.ly/alfa_Cronbach. 